\title{
Discrepancy Among Observational Studies: Example of Naproxen- Associated Adverse Events
}

\author{
Elham Rahme ${ }^{*, 1,2}$, Jean-Philippe Lafrance ${ }^{3}$, Hacene Nedjar ${ }^{2}$, Gilbert Rahme ${ }^{2}$ and Suzanne Morin ${ }^{1,2}$ \\ ${ }^{I}$ Department of Medicine, McGill University, Montreal, Canada and ${ }^{2}$ Research Institute, McGill University Health \\ Centre, Montreal, QC, Canada \\ ${ }^{3}$ Department of Epidemiology, Biostatistics and Occupational Health, McGill University, Montreal, QC, Canada
}

\begin{abstract}
Background: Observational studies assessing the cardiovascular adverse effect of naproxen have had conflicting results. It is not clear whether variation in population characteristics between studies may explain some of this discrepancy.

Objective: To determine whether changes in patient characteristics of naproxen users occurred between 1999 and 2004 in Québec, Canada and to examine whether these temporal changes were accompanied by changes in estimates of naproxenrelated hospitalizations for gastrointestinal (GI) ulcers and myocardial infarction, using provincial health services administrative databases.

Methods: Demographic, pharmaceutical and physician billing records of patients 65 years and older, who received naproxen or acetaminophen prescriptions between 1999 and 2004 were used. Two identical cohort studies, labeled Study 1 and Study 2 were conducted and their results were compared. One study was confined to the time period 1999-2001 and the other to 2002-2004. Patient characteristics at index date (the date of the first naproxen or acetaminophen prescription during the corresponding period) were compared between the study cohorts in naproxen and acetaminophen users, respectively, and within each study cohort between naproxen and acetaminophen users, using logistic regression models. Cox regression models with time dependent exposure were used to assess the association between naproxen $v s$ acetaminophen and hospitalizations for GI events or AMI, respectively within each study. Results were then compared between the two studies.

Results: Study 1 (1999-2001) cohort included 240,568 patients (205,238 acetaminophen and 35,330 naproxen) and Study 2 (2002-2004) cohort included 213,802 patients (193,918 acetaminophen and 19,884 naproxen). Patient characteristics of naproxen and acetaminophen users differed between the two studies. Naproxen users in Study 2 vs Study 1 were slightly younger, less likely to be females, less likely to have concomitant GI disease, less likely to have osteoarthritis and other co-morbidities and more likely to have used proton pump inhibitors, antihypertensive agents, anticoagulants, clopidogrel and aspirin. In general, similar changes in patient characteristics were observed in acetaminophen users between the two study cohorts. Compared to acetaminophen (without aspirin), the estimates of the GI risks with naproxen whether, used with or without aspirin, were significantly higher in Study 2 vs Study 1 [Hazard Ratio (HR) (95\% CI): 4.94 (3.48, 7.02)] vs $[2.22(1.62,3.06)]$, naproxen with aspirin [4.94 $(2.93,8.33)$ vs $2.47(1.48,4.12)]$, and acetaminophen and aspirin: [2.31 $(1.89,2.82)$ vs $1.46(1.20,1.77)]$. The estimate of the AMI risk with naproxen also seemed to be higher in Study $2 v s$ Study 1, however the increase was not statistically significant [HR $(95 \% \mathrm{CI})$ in the naproxen group: $1.18(0.83,1.67)$ in Study 1 vs $0.94(0.70,1.25)$ in Study 2], naproxen with aspirin. [1.44 $(0.95,2.18)$ vs $1.05(0.68,1.61)]$; and acetaminophen and aspirin. $1.15(1.01,1.30)$ vs $1.10(0.97,1.26)$.

Conclusion: Variation in patient characteristics in naproxen users was observed between 1999 and 2004. This variation was likely to be accompanied by a variation in patient pre-disposition to GI events that may explain the increase in estimates of naproxen-related GI adverse events observed during this period.
\end{abstract}

\section{INTRODUCTION}

Traditional non-steroidal anti-inflammatory drugs (tNSAIDs) effectively relieve acute and chronic pain and reduce inflammation [1-3]. However, important gastrointestinal (GI) and cardiovascular (CV) adverse events can occur with the use of these drugs [4-6]. The association between tNSAIDS

*Address correspondence to this author at the McGill University Health Centre, Division of Clinical Epidemiology, 687 Pine Avenue West, V Building, Montreal, Québec, H3A 1A1, Canada; Tel: (514) 934-1934, Ext. 44724; Fax: (514) 934-8293; E-mail: elham.rahme@mcgill.ca and GI adverse events is well established [7]. Serious tNSAID-associated GI adverse events, including gastroduodenal erosions and life-threatening complications such as bleeding and perforation in the upper GI tract are hard to predict, since they are not often preceded by warning symptoms [8]. To prevent these complications, it is recommended to use a gastroprotective agent (GPA) [misoprostol, histamine $_{2}$-receptor antagonists $\left(\mathrm{H}_{2} \mathrm{RAs}\right)$, or proton pump inhibitor (PPI)] with tNSAIDs $[9,10]$. The prescription of GPA with tNSAID reduces the risk of GI adverse events associated with these medications, but does not eliminate it and considerably increases patient management cost [11]. Cy- 
clooxygenase (COX)-2 selective NSAIDs have been shown to have an improved GI safety profile as compared to tNSAIDs [4, 12]. However, some clinical trials have found that some COX-2 selective NSAIDs may have serious CV adverse events [4, 13-15]. Some studies have also suggested that the $\mathrm{CV}$ risk is not unique to COX-2 selective NSAIDs, but may be common to all NSAIDs [16]. Naproxen is among the tNSAIDs that are frequently used in Québec [17]. At least one clinical trial has found that naproxen may have an improved CV safety profile over some COX-2 selective NSAIDs [4]. Patients in this trial were not allowed to use aspirin and it was suggested that the use of aspirin with COX-2 inhibitors in the patients at risk of CV adverse events may decrease this risk [4]. Clinical trials, specifically designed to study the $\mathrm{CV}$ adverse events with naproxen or with the combination of naproxen and aspirin, are lacking. The health care community has relied on observational studies to assess these events in real life settings. Several studies have been conducted in various countries and provinces, but results are conflicting [18-20]. Reasons explaining differences in results between studies have not been delineated. The characteristics of patients using naproxen in clinical practice may differ substantially from those of patients that are included in clinical trials from which sick patients are usually excluded. These characteristics may also differ between countries and between provinces or states within the same country, because of different drug regulation policy or marketing strategies. The characteristics of the population using a certain medication may also change overtime within the same province or state, when knowledge about the medication changes or when a new medication is marketed.

We used health services administrative databases to determine whether changes in patient characteristics occurred among naproxen and acetaminophen users in 2002-2004 vs 1999-2001 in Québec, Canada, and to examine whether these temporal changes were accompanied by changes in estimates of naproxen $v s$ acetaminophen-related hospitalizations for GI events and acute myocardial infarction (AMI).

\section{METHODS}

\section{Data Sources}

Data for this study were obtained from the Québec health services administrative databases administered by the Régie de l'assurance maladie du Québec (RAMQ). In Québec, Canada, all persons aged 65 years or older, who received social assistance, and those who do not have private drug insurance through their employer since 1997 are eligible for coverage, under the drug reimbursement program administered by RAMQ. The RAMQ prescription drugs database contains information on all outpatient dispensed prescriptions, including drug name, dispensation date, dosage, form, duration, and quantity of the drug dispensed. Drugs dispensed to patients during stays in the hospitals or public nursing homes, and over-the-counter drugs are not included in the database. Coverage for outpatient and inpatient physician services is universal. The RAMQ physician claims databases contained information on physician encounters, including date of the visit, place (outpatient clinic, or hospital), encrypted identification number of the physician, specialty of the physician, procedure performed (e.g. endoscopy) and diagnosis coded, using the International Classification of
Diseases, $9^{\text {th }}$ Revision (ICD-9). The Québec hospital discharge summary database provides information on all hospital admissions for the entire province. This information includes primary and up to 15 secondary diagnoses (coded according to the ICD-9 codes during the study period), admission and discharge dates, and procedures done. These databases are linkable through a unique patient identifier. Naproxen, aspirin and acetaminophen were available during the study period to all covered patients without any restriction to their use. Acetaminophen and aspirin were also available over the counter.

Permission from the Government of Québec ethics committee, the Commission d'accès à l'information, was obtained to use the data.

\section{Design and Data}

We conducted and compared the results of two separate population-based retrospective cohort studies, using the same design, methodology and data source, but covering two different time periods, January 1999 -December 2001 (Study 1) and January 2002- December 2004 (Study 2), respectively. In both studies, we used RAMQ and hospital admission records of patients aged 65 years or older, who filled at least one prescription for naproxen or acetaminophen during the corresponding study period. Hospitalizations for upper GI events (ulcer, bleeding or perforation) and for AMI during that period were identified from the hospital discharge summary database.

\section{Inclusion/Exclusion Criteria}

Two cohorts, one for each study, were constructed in a similar manner. The date of the first filled prescription for naproxen or acetaminophen during each study period, respectively, was considered as the patient's index date for the corresponding cohort. New users in each cohort were defined as patients, who had not filled a prescription for the study medications in the year prior to the index date. Patients with a GI or AMI hospitalization, as defined below, in the year prior to the index date were excluded. Patients who filled prescriptions for both naproxen and another NSAID or naproxen and acetaminophen on the index date were also excluded.

\section{Outcome Measure}

The outcomes of interest were the first hospitalization for an upper GI event (ulcer, bleeding or perforation in the stomach or duodenum) or for AMI during the corresponding study period. Hospitalization was considered only for the patients who were discharged from acute care hospitals with GI event (ICD-9 codes: 531.x, 532.x, 533.x, 534.x, 578.x) or AMI (ICD-9 code 410.x), recorded as the most responsible diagnosis (primary discharge diagnosis).

\section{Follow-Up}

In each cohort, patients were followed from their index date to the earliest of: an upper GI hospitalization, an AMI hospitalization, death or the end of the corresponding study period.

\section{Drug Exposures}

Exposure to a study drug was defined as the number of days of medication supplied, as recorded in the database, in 
addition to a grace period of $25 \%$ of this number. A hospitalization for GI or AMI event that occurred during an exposure period was attributed to that period. Events that occurred during a period overlapping two study drugs were attributed to the drug dispensed last. This assumes that the patient stopped the use of the first drug at the date of the second drug dispensing. This assumption was checked in a sensitivity analysis. Exposure episodes, where two different study drugs were dispensed on the same day were excluded from the analyses. The use of aspirin may affect both GI and $\mathrm{CV}$ adverse events, as we had found in a previous study that the utilization of aspirin increased significantly among NSAID and acetaminophen users between 1999 and 2004 [21], we separated exposure episodes into those with and those without aspirin. Therefore, exposure episodes were classified into four categories: naproxen and aspirin, acetaminophen and aspirin, naproxen alone, and acetaminophen alone. A prescription of acetaminophen was classified as acetaminophen and aspirin, if the patient filled an aspirin prescription, which supplied days overlapped those for acetaminophen. The naproxen and aspirin category was defined in a similar manner. Patients in these categories were termed "users of aspirin". All exposure episodes occurred during follow-up were considered in the analyses. Therefore, patients could contribute time to more than one exposure category.

\section{Patient Characteristics}

Patient characteristics that were assessed at the index date were: age and gender; prescription for NSAIDs, use of GPA, antihypertensive agents, antidiabetic agents, lipid lowering agents or vasodilators in the prior year; prescriptions for corticosteroids, anticoagulants, clopidogrel or aspirin in the prior three months; GI events in the prior year (upper GI investigations (endoscopy, Barium swallow) and diagnoses of ulcer in the upper GI tract), ischemic heart disease, congestive heart failure, cerebrovascular disease, peripheral vascular disease, chronic or acute renal failure, anemia or blood diseases, chronic obstructive pulmonary disease (COPD), and osteoarthritis or rheumatoid arthritis.

\section{Statistical Analyses}

Patient characteristics at the index date were compared between naproxen and acetaminophen patients within each cohort, respectively, and between the two cohorts within naproxen and acetaminophen patients, respectively, using logistic regression models. In each study, the GI and AMI risks with naproxen used either with or without concomitant aspirin were compared to those with acetaminophen (without concomitant aspirin), using multivariable Cox regression models with time-dependent exposures and fixed baseline patient characteristics. Times of non-exposure were not included in the model to facilitate the analysis. Discontinuous time intervals removed the subjects from the risk sets during the time of non-exposure [22]. Sensitivity and sub-group analyses were conducted in each study. In the sensitivity analysis, events that occurred during overlapping times were attributed to the drug dispensed first and in sub-group analyses all analyses were repeated considering only 'New users'. All statistical analyses were performed using SAS for Linux, version 9.2 (SAS Institute Inc., Cary, NC, USA).

\section{RESULTS}

\section{Patient Characteristics at Index Date}

In Study 1 (1999-2001), the cohort included 240,568 patients (205,238 acetaminophen and 35,330 naproxen), and in Study 2 (2002-2004), the cohort included 213,802 patients (193,918 acetaminophen and 19,884 naproxen). Patient characteristics are displayed in Table $\mathbf{1}$ by cohort and study drug used at index date. Results of the logistic regression models, comparing patient characteristics between cohorts within users of each study drug, are also displayed in Table 1. As compared to patients who used naproxen in 19992001 , those who used it in 2002-2004 were $16 \%$ less likely to be females, $38 \%$ less likely to have had an outpatient diagnosis of GI ulcer, but $75 \%$ more likely to have used a PPI at the index date. They were also more likely to have filled prescriptions for PPIs (93\%), antihypertensive agents (37\%), anticoagulants $(47 \%)$, clopidogrel $(79 \%)$, and aspirin $(57 \%)$ in the prior year; less likely to have had a diagnosis with osteoarthritis (27\%), and CV diseases or COPD in the prior year (Table 1). In general, similar differences were observed among acetaminophen users across the two cohorts (Table 1). Table 2 displays the results of the logistic regression models comparing patient characteristics between acetaminophen and naproxen users within each cohort among all patients and among new users, respectively. In both cohorts, patients using naproxen were younger, included fewer females, had less GI or CV diseases and more of them had a diagnosis of arthritis.

\section{Naproxen, Acetaminophen and Aspirin Utilization in Follow-Up}

The total number of prescriptions filled by study patients during follow-up and the number of patient-years (p-years) of exposure from each exposure category are reported in Table 3. Patients in the first cohort filled 1,223,017 prescriptions for acetaminophen (only), 549,497 prescriptions for acetaminophen and aspirin, 134,991 prescriptions for naproxen (only), and 34,008 prescriptions for naproxen and aspirin, while patients in the second cohort filled 1,238,624 prescriptions for acetaminophen (only), 826,057 prescriptions for acetaminophen and aspirin, 90,045 prescriptions for naproxen (only), and 34,537 prescriptions for naproxen and aspirin.

\section{Hospitalizations for Gastrointestinal Events}

Unadjusted rates of GI hospitalizations seemed to be higher for naproxen users in Study 2 vs Study $1(6.57 / 1000$ p-years in 1999-2001 and 8.28/1000 p-years in 2002-2004) and lower in acetaminophen users $(4.46 / 1000$ p-years and $3.07 / 1000$ p-years, respectively) (Table 3). Results from the time-dependent Cox regression models, comparing the adjusted hazard ratios (HR) of GI hospitalization between the exposure categories are shown in Table 4. Compared to patients using acetaminophen (without aspirin), the adjusted HR [95\% confidence interval (CI)] of hospitalization for upper GI events was higher in Study 2 vs Study 1 among users of naproxen without aspirin 4.94 (3.48, 7.02) vs 2.22 $(1.62,3.06)$. This difference was statistically significant as evidenced by the non-overlapping confidence intervals. The 
Table 1. Comparison of Patient Characteristics at Index Dates Between Study 2 vs Study 1 Cohorts Using Logistic Regression Models

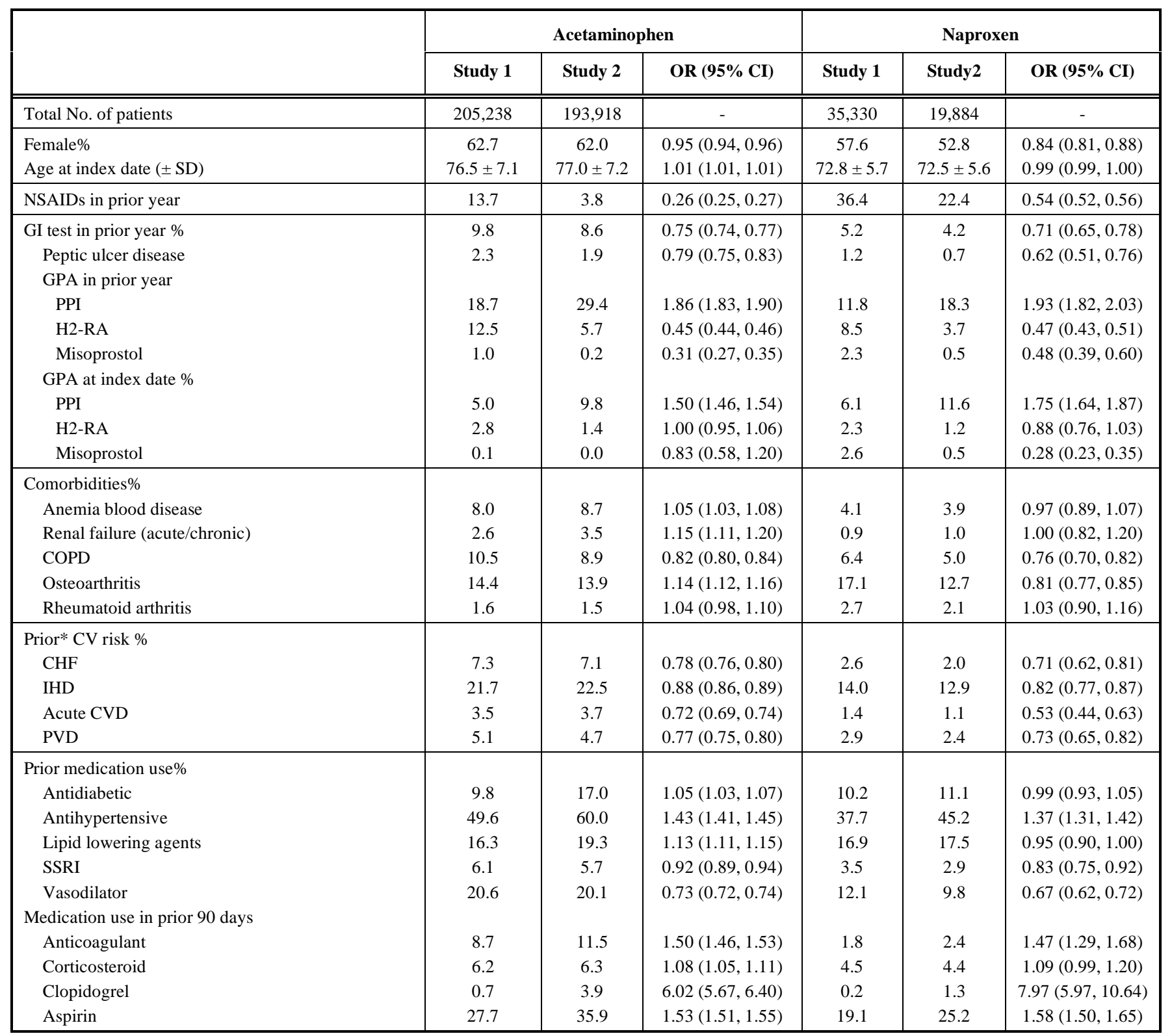

*Identified in the prior year.

Abbreviations: $\mathrm{GI}=$ gastrointestinal; $\mathrm{GPA}=$ gastroprotective agent; $\mathrm{PPI}=$ proton pump inhibitor; $\mathrm{H} 2 \mathrm{RA}=$ histamine- 2 receptor antagonist; $\mathrm{SSRI}=$ serotonin reuptake inhibitor; $\mathrm{COPD}=$ chronic obstructive pulmonary disease; $\mathrm{CHF}=$ congestive heart failure; $\mathrm{IHD}=$ ischemic heart disease; $\mathrm{CVD}=$ cerebrovascular disease; $\mathrm{PVD}=$ peripheral vascular disease; $\mathrm{CV}=$ cardiovascular disease; $\mathrm{OR}=$ odds ratio; $\mathrm{CI}=$ confidence interval; $\mathrm{SD}=$ standard deviation.

HR of GI hospitalizations was also significantly higher in Study 2 vs Study 1 among users of acetaminophen with aspirin $2.31(1.89,2.82)$ vs $1.46(1.20,1.77)$, but not among users of naproxen with aspirin $4.49(2.93,8.33)$ vs 2.47 (1.48, 4.12 ), although results pointed to that direction.

\section{Hospitalizations for Acute Myocardial Infarction}

Unadjusted rates of hospitalizations for AMI seemed to be similar in Study 2 vs Study 1 among naproxen users, although results pointed to a possible small increase in Study 2 (Table 3). Results from the time-dependent Cox regression models, comparing the adjusted HR of AMI hospitalizations between the exposure categories are shown in Table 4. Compared to patients using acetaminophen (without aspirin), the adjusted HR (95\% CI) of AMI hospitalization was also not statistically different between Study 2 and Study 1 in all other exposure categories, although results pointed to a possible small increase in Study 2 (Table 4).

Sensitivity analyses and subgroup analyses conducted in new users revealed similar results (data not shown).

\section{DISCUSSION}

This study compared the estimates of the risks of hospitalizations for upper GI events and AMI among naproxen and acetaminophen users, obtained from two identical studies conducted separately in the same geographical area over two different time-periods. Patient characteristics of users of these medications differed between studies and the risks of GI hospitalizations among naproxen $v s$ acetaminophen users seemed to be higher in Study 2 vs Study 1. As we used the 
Table 2. Logistic Regression Models Comparing Patient Characteristics of those Using Naproxen vs Acetaminophen at the Index Date in the Two Study Cohorts

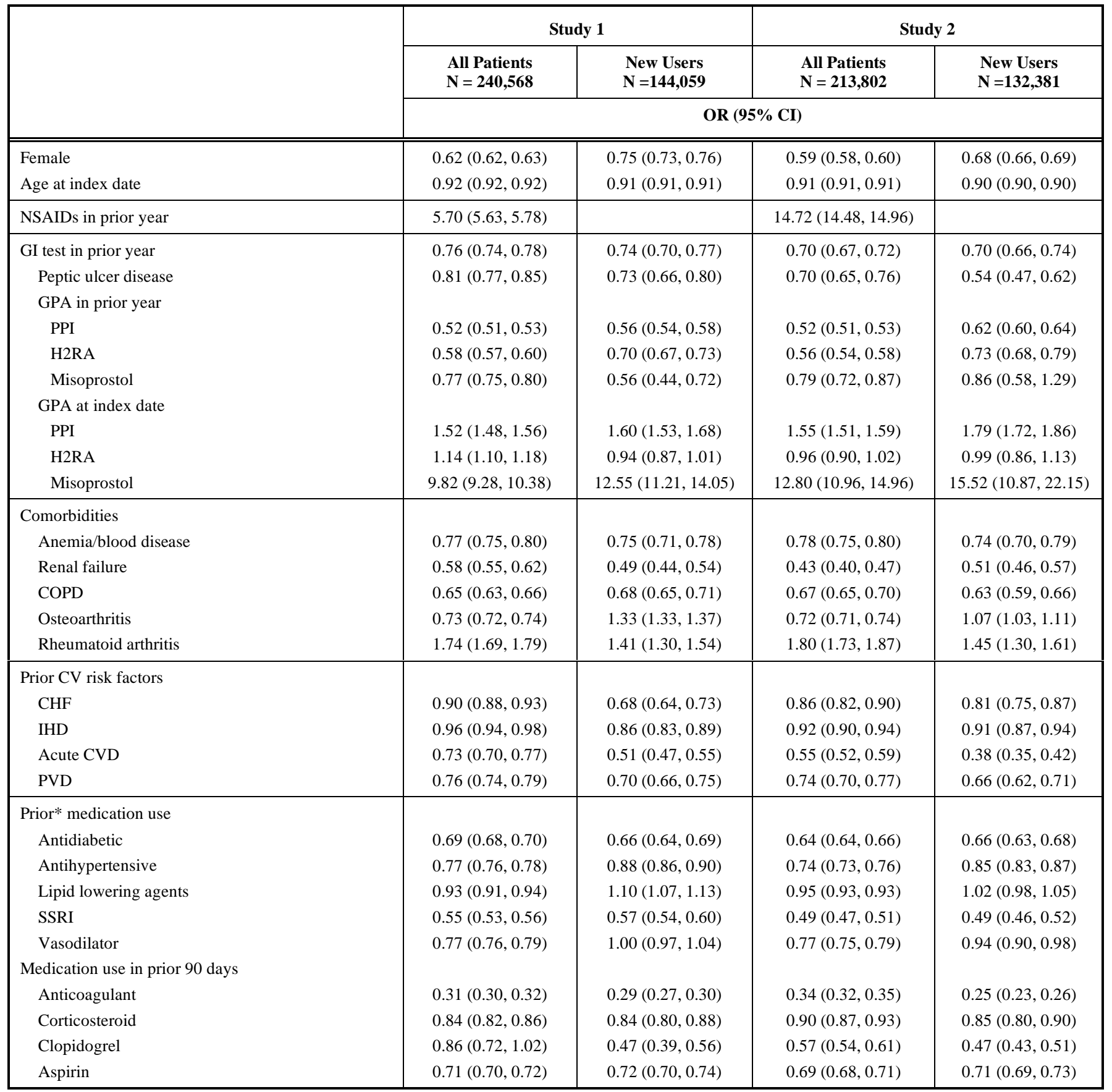

*In prior year.

same methodology, adjusted for all known confounders that could be measured from the database in both studies, we hypothesized that the increase in risk estimates observed in Study 2 among naproxen users is likely to be attributable to a higher pre-disposition to GI events of naproxen users in that study and/or a lower pre-disposition in acetaminophen users. This could be possible, if for example more patients at higher risk of GI events used naproxen in the second period, because of a perception of a better AMI safety with the drug and/or if more patients at high risk of GI events switched from acetaminophen to a COX-2 inhibitor, however, these hypotheses cannot be confirmed from the current data.
Nonetheless, these results suggest that estimates from observational studies assessing drug-related adverse events are affected by differences in study patients' characteristics, in particular those that cannot be measured and for adjusted in multivariable models such as disease severity and predisposition to the adverse event.

Published studies assessing the association between naproxen use and GI events have found an increased risk with naproxen either when compared to no-use [27], acetaminophen [28] or other NSAIDs, [29, 30] however, the magnitude of this increase has varied between the studies [27, 31, 32]; some reporting a two-fold increase [27], while others reporting a 9-fold increase [31]. In our study, we 
Table 3. Total Number of Prescriptions and Crude Rates of GI and AMI Hospitalizations

\begin{tabular}{|c|c|c|c|c|c|}
\hline & $\begin{array}{l}\text { No. of } \\
\text { Patients }\end{array}$ & $\begin{array}{c}\text { No. of } \\
\text { Prescriptions }\end{array}$ & $\begin{array}{l}\text { Total Dura- } \\
\text { tion (Years) }\end{array}$ & $\begin{array}{l}\text { No. of GI Hospitalizations } \\
\text { (Rate /1000 p-Years) }\end{array}$ & $\begin{array}{l}\text { No. of AMI Hospitalizations } \\
\text { (Rate /1000 p-Years) }\end{array}$ \\
\hline \multicolumn{6}{|c|}{ Study 1} \\
\hline Acetamin & 153,638 & $1,223,017$ & 62,974 & $281(4.46)$ & $585(9.28)$ \\
\hline Naproxen & 29,056 & 134,991 & 7,904 & $49(6.20)$ & $52(6.57)$ \\
\hline Naproxen + aspirin & 6,274 & 34,008 & 1,957 & $16(8.17)$ & $22(11.24)$ \\
\hline \multicolumn{6}{|c|}{ Study 2} \\
\hline Acetamin & 129,429 & $1,238,624$ & 62,826 & $193(3.07)$ & $559(8.89)$ \\
\hline Naproxen & 15,200 & 90,045 & 5,191 & $43(8.28)$ & $35(6.74)$ \\
\hline
\end{tabular}

Abbreviations: yrs= years; $\mathrm{p}$-yrs=patient-years; $\mathrm{GI}=$ gastrointestinal; $\mathrm{AMI}=$ acute myocardial infarction .

Table 4. Association Between Naproxen vs Acetaminophen Utilisation and Hospitalization for GI and AMI Adverse Events- CoxRegression Models with Time Dependent Exposure

\begin{tabular}{|l|c|c|c|c|}
\hline \multirow{2}{*}{} & \multicolumn{2}{|c|}{ AMI Hospitalizations } & \multicolumn{2}{c|}{ GI Hospitalizations } \\
\cline { 2 - 6 } & Study 1 & Study 2 & \multicolumn{2}{c|}{ Study 1 } \\
\hline \hline \multicolumn{3}{|c|}{ HR (95\% CI) } \\
\hline Acetamin. & 1 (reference) & 1 (reference) & $2.22(1.62,3.06)$ & 1 (reference) \\
\hline Naproxen & $0.94(0.70,1.25)$ & $1.18(0.83,1.67)$ & $1.46(1.20,1.77)$ & $2.31(1.89,2.82)$ \\
\hline Acetamin.+ aspirin & $1.10(0.97,1.26)$ & $1.15(1.01,1.30)$ & $2.47,7.02)$ \\
\hline Naproxen + aspirin & $1.05(0.68,1.61)$ & $1.44(0.95,2.18)$ & $4.48,4.12)$ & $4.94(2.93,8.33)$ \\
\hline
\end{tabular}

The models adjusted for all patient characteristics that appear in Table $\mathbf{1}$.

reporting a 9-fold increase [31]. In our study, we found a acetaminophen users in Study 1, and a 5-fold increase in Study 2. Observational studies assessing the association between naproxen and CV adverse events have also had conflicting results [16, 18, 20, 23-26]. Many of these studies have found a beneficial $\mathrm{CV}$ effect of naproxen when compared to other NSAIDs [16, 18, 23], while others did not find such effect when naproxen was compared to no or remote use of NSAID, $[25,26]$ with some finding an increased risk with naproxen $[20,24]$.

While many studies have discussed the possible presence of channeling bias occurring when higher risk patients are more likely to be prescribed a specific drug [33, 34], and methods to reduce this bias have been recommended; [34] no previously published study has documented the magnitude of variation in results over time in the same population. Recently, one paper investigating the risk of AMI with NSAIDs, showed that adjustments for the dose and pattern of utilization of the medications (number of previous prescriptions, duration, and number of gaps between previous prescriptions) significantly affect the results [35]. In our analyses, results between the two studies remained different, when we considered only 'New users' (those who did not have any NSAID or acetaminophen prescription in the previous year) (data not shown). Therefore, previous use of NSAID cannot solely account for the difference in results observed between the two studies.

Our two studies were conducted using large, populationbased, well-validated medical databases that included patients with multiple GI and AMI risk factors who are typically excluded from clinical trials. We used identical designs and methods in both studies to rule out the possibility of any change related to the methodology used. Nonetheless, our studies have limitations. First, misclassification may have occurred in the identification of some co-morbidity that relied on physician diagnoses such as COPD, renal failure, alcohol or drug abuse, osteoarthritis, and rheumatoid arthritis. However, these misclassifications, if they occurred, were likely to be non-differential between studies and between study medications within each study and are unlikely responsible for the important change in results observed between the two studies. Second, differences may exist between patients prescribed acetaminophen and those prescribed naproxen, based on variables that were not available in the 
database such as use of non-prescription aspirin or ibuprofen and a history of GI events prior to the period examined in this study, however, these variables are not expected to differ between the two studies and although there is a possibility that they may have biased the results in each study toward the null, they were unlikely responsible for the change in HR observed between the two studies.

In conclusion, our study shows that estimates of the risk of medication-related adverse events may differ with differences in study populations.

\section{ACKNOWLEDGEMENT}

Dr. Rahme had full access to all of the data in the study and takes responsibility for the integrity of the data and the accuracy of the data analysis.

\section{STATEMENT OF INTERESTS}

\section{Authors' Declaration of Personal Interests}

Dr. Morin has received honoraria (consultant and speaker fees) from the Procter and Gamble, sanofiaventis, Amgen and Novartis in the course of other studies.

(ii) Dr. Rahme has received research funding and consultant fees from Merck \& Co. Inc., Pfizer Canada, Boehringer Ingelheim, Chiesti Farmaceutici and ETHYPHARM in the course of other studies. All other authors have no conflict of interest.

\section{REFERENCES}

[1] Wolfe F, Zhao S, Lane N. Preference for nonsteroidal antiinflammatory drugs over acetaminophen by rheumatic disease patients: a survey of 1,799 patients with osteoarthritis, rheumatoid arthritis, and fibromyalgia. Arthritis Rheum 2000; 43: 378-85.

[2] Pincus T, Koch GG, Sokka T, et al. A randomized, double-blind, crossover clinical trial of diclofenac plus misoprostol versus acetaminophen in patients with osteoarthritis of the hip or knee. Arthritis Rheum 2001; 44(7), 1477-80.

[3] Laine L, White WB, Rostom A, Hochberg M. COX-2 Selective Inhibitors in the treatment of osteoarthritis. Semin Arthritis Rheum 2008 3; 38: 165-87.

[4] Bombardier C, Laine L, Reicin A, et al. Comparison of upper gastrointestinal toxicity of rofecoxib and naproxen in patients with rheumatoid arthritis. VIGOR Study Group. N Engl J Med 2000; 343: $1520-8$.

[5] Silverstein FE, Faich G, Goldstein JL, et al. Gastrointestinal toxicity with celecoxib vs nonsteroidal anti-inflammatory drugs for osteoarthritis and rheumatoid arthritis: the CLASS study: A randomized controlled trial. celecoxib long-term arthritis safety study. JAMA 2000; 284: 1247-55.

[6] Coruzzi G, Venturi N, Spaggiari S. Gastrointestinal safety of novel nonsteroidal antiinflammatory drugs: selective COX-2 inhibitors and beyond. Acta Biomed 2007; 78: 96-110.

[7] Wolfe MM, Lichtenstein DR, Singh G. Gastrointestinal toxicity of nonsteroidal antiinflammatory drugs. N Engl J Med 1999; 340: 1888-99.

[8] Barrison AF, Wolfe MM. Management of NSAID-related gastrointestinal mucosal injury. Inflammopharmacology 1999; 7: 277-86.

[9] American College of Rheumatology. Update of ACR guidelines for osteoarthritis: role of the coxibs. J Pain Symptom Manage 2002; 32(4 Suppl): S31-S34.

[10] Scheiman JM, Yeomans ND, Talley NJ, et al. Prevention of ulcers by esomeprazole in at-risk patients using non-selective NSAIDs and COX-2 inhibitors. Am J Gastroenterol 2006; 101: 701-10.

[11] Rahme E, Toubouti Y, LeLorier J. Utilization and cost comparison of current and optimal prescribing of nonsteroidal antiinflammatory drugs in Quebec, Canada. J Rheumatol 2006; 33: 588-96.
[12] Goldstein JL, Correa P, Zhao WW, et al. Reduced incidence of gastroduodenal ulcers with celecoxib, a novel cyclooxygenase-2 inhibitor, compared to naproxen in patients with arthritis. Am J Gastroenterol 2001; 96: 1019-27.

[13] Bresalier RS, Sandler RS, Quan H, et al. Cardiovascular events associated with rofecoxib in a colorectal adenoma chemoprevention trial. N Engl J Med 2005; 352: 1092-102.

[14] Nussmeier NA, Whelton AA, Brown MT, et al. Complications of the COX-2 inhibitors parecoxib and valdecoxib after cardiac surgery. N Engl J Med 2005; 352: 1081-91.

[15] Solomon SD, McMurray JJV, Pfeffer MA, et al. Cardiovascular risk associated with celecoxib in a clinical trial for colorectal adenoma prevention. N Engl J Med 2005; 352: 1071-80.

[16] McGettigan P, Henry D. Cardiovascular risk and inhibition of cyclooxygenase: a systematic review of the observational studies of selective and nonselective inhibitors of cyclooxygenase 2. JAMA 2006; 296: 1633-44.

[17] Rahme E, Nedjar H, Bizzi A, Morin S. Hospitalization for gastrointestinal adverse events attributable to the use of low-dose aspirin among seniors also using NSAIDs: a retrospective cohort study. Aliment Pharmacol Ther 2007; 26: 1387-98.

[18] Rahme E, Pilote L, LeLorier J. Association between naproxen use and protection against acute myocardial infarction. Arch Int Med 2002; 162: 1111-5.

[19] Watson DJ, Rhodes T, Cai B, Guess HA. Lower risk of thromboembolic cardiovascular events with naproxen among patients with rheumatoid arthritis. Arch Intern Med 2002; 162: 1105-10.

[20] Graham DJ, Campen D, Hui R, et al. Risk of acute myocardial infarction and sudden cardiac death in patients treated with cyclooxygenase 2 selective and non-selective non-steroidal antiinflammatory drugs: nested case-control study. Lancet 2005; 365 : 475-81.

[21] Rahme E, Nedjar H, Bizzi A, Morin S. Hospitalization for gastrointestinal adverse events attributable to the use of low-dose aspirin among patients 50 years or older also using non-steroidal antiinflammatory drugs: a retrospective cohort study. Aliment Pharmacol Ther 2007; 26: 1387-98.

[22] Ake CF, Carpenter AL. Survival Analysis with PHREG: Using MI and MIANALYZE to Accomodate Missing Data. 2002.

[23] Hippisley-Cox J, Coupland C, Logan R. Risk of adverse gastrointestinal outcomes in patients taking cyclo-oxygenase- 2 inhibitors or conventional non-steroidal anti-inflammatory drugs: population based nested case-control analysis. BMJ 2005; 331: 1310-6.

[24] Rahme E, Nedjar H. Risks and benefits of COX-2 inhibitors vs non-selective NSAIDs: does their cardiovascular risk exceed their gastrointestinal benefit? A retrospective cohort study. Rheumatology (Oxford) 2007; 46: 435-8.

[25] Ashworth NL, Peloso PM, Muhajarine N, Stang M. Risk of hospitalization with peptic ulcer disease or gastrointestinal hemorrhage associated with nabumetone, Arthrotec, diclofenac, and naproxen in a population based cohort study. J Rheumatol 2005; 32: 2212-7.

[26] Strom BL, Schinnar R, Bilker WB, Feldman H, Farrar JT, Carson JL. Gastrointestinal tract bleeding associated with naproxen sodium vs ibuprofen. Arch Intern Med 1997; 157: 2626-31.

[27] Lewis SC, Langman MJ, Laporte JR, Matthews JN, Rawlins MD, Wiholm BE. Dose-response relationships between individual nonaspirin nonsteroidal anti-inflammatory drugs (NANSAIDs) and serious upper gastrointestinal bleeding: a meta-analysis based on individual patient data. Br J Clin Pharmacol 2002; 54: 320-6.

[28] Henry D, Lim LL, Garcia Rodriguez LA, et al. Variability in risk of gastrointestinal complications with individual non-steroidal antiinflammatory drugs: results of a collaborative meta-analysis. BMJ 1996; 312: 1563-6.

[29] Solomon DH, Glynn RJ, Levin R, Avorn J. Nonsteroidal antiinflammatory drug use and acute myocardial infarction. Arch Intern Med 2002; 162: 1099-104.

[30] Levesque LE, Brophy JM, Zhang B. The risk for myocardial infarction with cyclooxygenase- 2 inhibitors: a population study of elderly adults. Ann Intern Med 2005; 142: 481-9.

[31] Mamdani M, Rochon P, Juurlink DN, et al. Effect of selective cyclooxygenase 2 inhibitors and naproxen on short-term risk of acute myocardial infarction in the elderly. Arch Int Med 2003; 163: 481-6. 
[32] Ray WA, Stein CM, Hall K, Daugherty JR, Griffin MR. Nonsteroidal anti-inflammatory drugs and risk of serious coronary heart disease: an observational cohort study. Lancet 2002; 359: 118-23.

[33] MacDonald TM, Pettitt D, Lee FH, Schwartz JS. Channelling of patients taking NSAIDs or cyclooxygenase-2-specific inhibitors and its effect on interpretation of outcomes. Rheumatol 2003; 42: 3-10.
[34] Morant SV, Pettitt D, MacDonald TM, Burke TA, Goldstein JL. Application of a propensity score to adjust for channelling bias with NSAIDs. Pharmacoepidemiol Drug Saf 2004; 13: 345-53.

[35] Van Staa TP, Rietbrock S, Setakis E, Leufkens HG. Does the varied use of NSAIDs explain the differences in the risk of myocardial infarction? J Intern Med 2008; 264(5): 481-92.

(C) Rahme et al.; Licensee Bentham Open.

This is an open access article licensed under the terms of the Creative Commons Attribution Non-Commercial License (http://creativecommons.org/licenses/by$\mathrm{nc} / 3.0 /$ ) which permits unrestricted, non-commercial use, distribution and reproduction in any medium, provided the work is properly cited. 